\title{
Wireless Power Transmission for Mobile and Vehicle
}

\author{
Vithyaa.M ${ }^{1}$, Marthandan. $\mathrm{R}^{2}$ \\ M.ECommunication systems Adhiyamaan college of engineering, Hosur,India \\ Asst professor dept of ece Adhiyaman college of engineering, Hosur,India
}

\begin{abstract}
Wireless Power Transfer Technology is an emerging trend in the world today. This technology eliminates the drawbacks of existing wired technology. In this project, wireless power transfer is used in charging the mobile phones of the passengers travelling in buses and battery of the bus. Rechargeable wireless chargers or wired mobile phone chargers cannot provide charge for mobile phones at all time at all places. It may be difficult to carry a wired charger with us when moving from one place to another. If the charge of their mobile phone becomes low during emergency situation they cannot search for plug points inside the vehicles. To overcome these drawbacks we implement wireless power transfer technology by which mobile phone and vehicle can be charged wirelessly. The working principle behind wireless power transmission is electromagnetic induction. The vehicle will be controlled by using switches in the vehicle. Wireless transmission is useful in cases where interconnecting wires are inconvenient, hazardous, or impossible.
\end{abstract}

Keywords: WPT Wireless Power Transfer (or) Transmission, Electromagnetic induction, Charging mobile phones

\section{Introduction:}

We need a power source and a transmitting antenna, and also a receiving antenna to which we can connect the thing to be powered (the electrical load, or just the load). The power source will deliver a high power signal to the antenna. This will create an electrostatic field around the antenna that changes as the signal to it changes. This will create electromagnetic waves that will travel out from the antenna and through the air. The receiving antenna will be in the path of these waves, and the waves will pass by it and "sweep" it with their moving electromagnetic fields. This will induce a signal in the receiving antenna proportional to the energy that the antenna captures. This signal will cause current flow that will power the load.

\section{a. ELECTROMAGNETIC INDUCTION:}

An electric current flowing through a conductor, such as a wire, carries electrical energy. When an electric current passes through a circuit there is an electric field in the dielectric surrounding the conductor; magnetic field lines around the conductor and lines of electric force radically about the conductor.

\section{b. PRINCIPLE OF ELECTROMAGNETIC INDUCTION}

"When a conductor is exposed to a changing magnetic field, an electric current will flow in the conductor."

\section{c. PROPOSED SYSTEM MODEL:}

In the proposed system, wireless power transmission technology is implemented to charge the mobile phones of users travelling in a bus. The wireless power transmission requires a coil at both the ends. The transmitter coil is connected to the plug point using a power chord. The incoming $\mathrm{AC}$ power from the plug point is first converted into $12 \mathrm{~V} \mathrm{AC}$ by using a step down transformer. Then the $12 \mathrm{~V} \mathrm{AC}$ will be converted into $12 \mathrm{~V}$ DC by using a Rectifier circuit. This $12 \mathrm{~V}$ DC signal will be passed to the transmitter coil to transmit it wirelessly. The receiver coil present within the receive range will receive the power wirelessly. The received $12 \mathrm{~V}$ power will then be stored in a rechargeable battery from which the wheel chair is operated. The $12 \mathrm{~V}$ signal is also reduced to $5 \mathrm{~V}$ DC signal which is the input voltage of the mobile phone and controller board. The reduced 5V signal will be sent to the mobile phone charger point to charge the mobile and the vehicle's battery. The switches in the vehicle are used to control its movements like forward, reverse, left and right. 

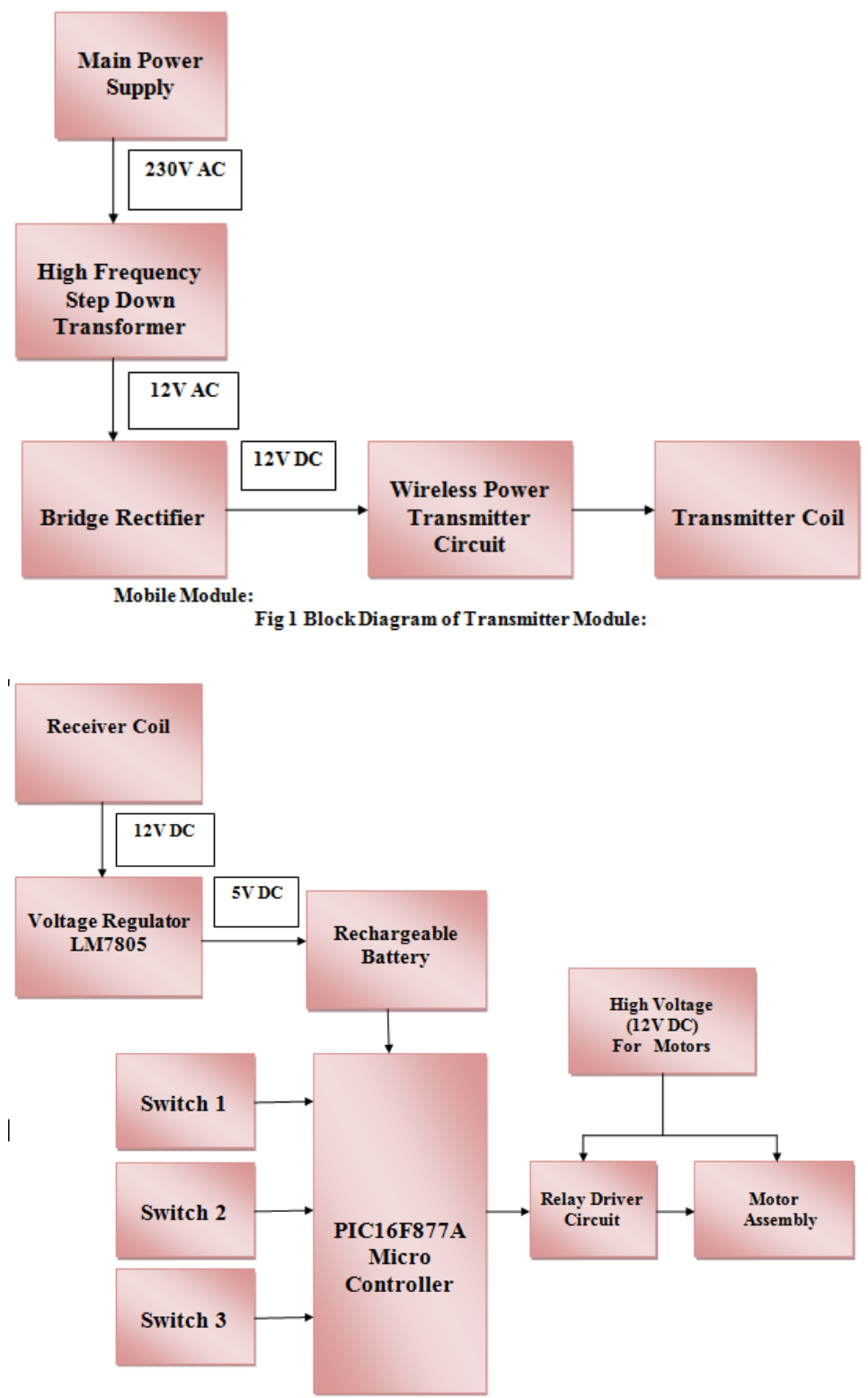

Fig 2 Block diagram of vehicle module

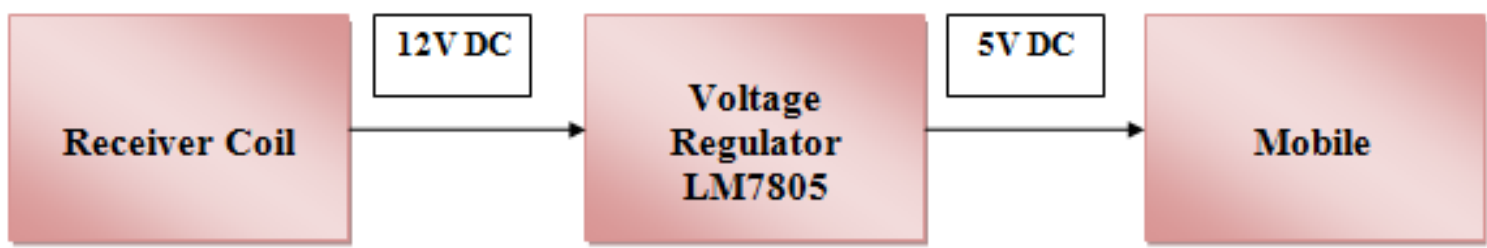

Fig3 block diagram of mobile module

\section{Needs Of Wireless Power Transfer}

The main function of WPT is to allow electrical devices to be continuously charged and lose the constrain of power cord. The main concept of WPT is to transfer electrical power from a source a device without the aid of wires. The technology behind wireless charging have been around for a long time the idea was initially suggested by Nikola Tesla, who demonstrated the principle of wireless charging it is already a reality in such devices as electric toothbrushes and surgically implanted devices, like artificial hearts.

Wireless charging, also known as inductive charging, is based on a few simple principles. The technology requires two coils: a transmitter and a receiver. An alternating current is passed through the 
transmitter coil, generating a magnetic field. This in turn induces a voltage in the receiver coil; this can be used to power a mobile device or charge a battery.

\section{Application Of Wireless Power Transfer}

The first applications that the charging station that will range from about one to five meters. This is a small box-like object that will be able to charge compatible electronics within the range of the system. Wireless power transfer charging systems are proven to have efficiencies near that of conventional charging devices. The range of the electromagnetic waves will begin to increase. Once the range reaches around twenty meters, entire homes will be able to be charged by a single transmitter located close to the home. As the range expands entire blocks and streets will be powered by a single transmitter.

\section{Merits Of Wireless Power Transfer}

a) Wireless Power Transmission system would completely eliminates the existing high-tension power transmission line cables, towers and sub stations

b) The power could be transmitted to the places where the wired transmission is not possible. Loss of transmission is negligible level in the Wireless Power Transmission; therefore, the efficiency of this method is very much higher than the wired transmission.

c) The power failure due to short circuit and fault on cables would never exist in the transmission and power theft would be not possible at all.

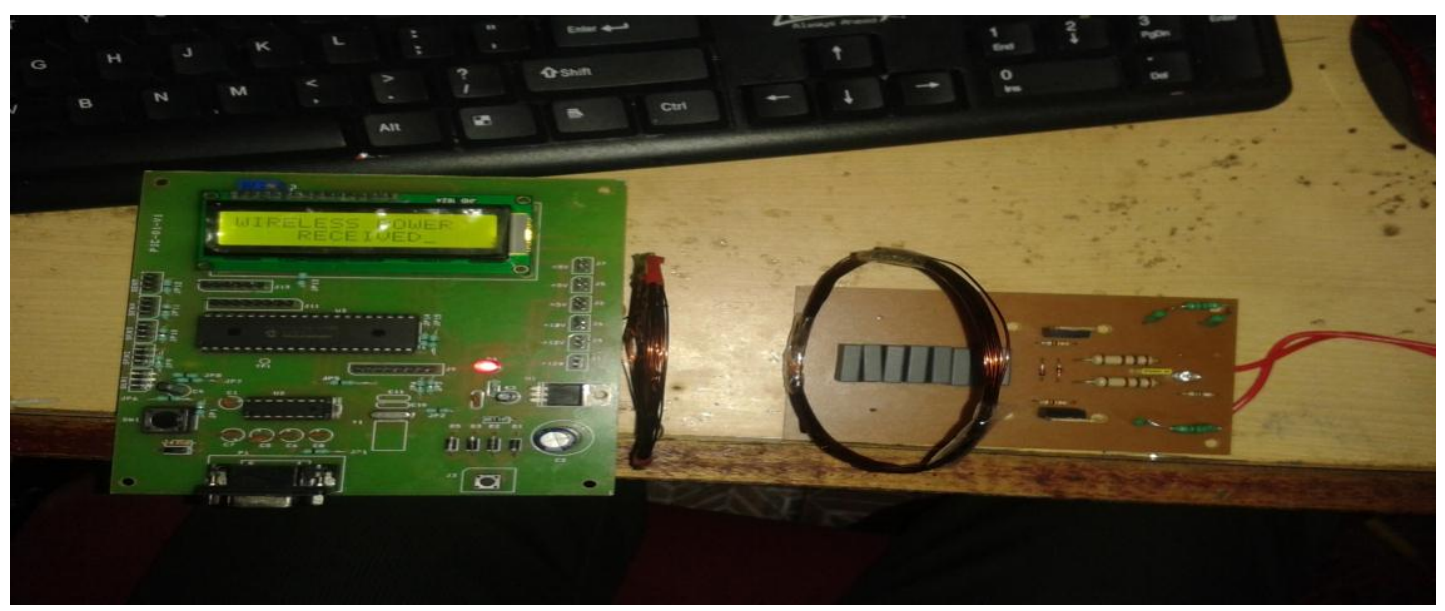

Fig 4 hardware output for wireless power transfer

\section{Conclusion}

This paper analyzed the wireless power transmission for a mobile, and explain the basic technique of transmitting power Wireless Power Transmission technology is a mandatory need in the present scenario of charging system in electronic accessories.

\section{Reference}

[1] Z. N. Low, R. A. Chinga, R. Tseng, and J. Lin, "Design and Test of a High-Power High-Efficiency Loosely Coupled Planar Wireless Power Transfer System," IEEE Transactions on Industrial Electronics, Vol. 56, No. 5, pp. 1801-1812, May 2009.

[2]. A.K. Ram Rakhyani, S. Mirabbasi, M. Chiao, 'Design and Optimization of Resonance-based Efficient Wireless Power Delivery Systems for Biomediacal Implants', IEEE Trans. On Biomediacal Circuits and Systems, Vol.5, No.1, Feb.2011.

[3]. J. A. Taylor, Z. N. Low, J. J. Casanova, J. Lin, "A Wireless Power Station for Laptop Computers," Proceedings of IEEE Radio and Wireless Symposium, pp. 625-628, January 2010.

[4]. http://en.wikipedia.org/wiki/wireless power transmission 